PROCEEDINGS OF THE

AMERICAN MATHEMATICAL SOCIETY

Volume 139, Number 6, June 2011, Pages 1915-1929

S 0002-9939(2010)10566-X

Article electronically published on November 2, 2010

\title{
CONGRUENCES CONCERNING LEGENDRE POLYNOMIALS
}

\author{
ZHI-HONG SUN
}

(Communicated by Ken Ono)

\begin{abstract}
Let $p$ be an odd prime. In this paper, by using the properties of Legendre polynomials we prove some congruences for $\sum_{k=0}^{\frac{p-1}{2}}\left(\begin{array}{c}2 k \\ k\end{array}\right)^{2} m^{-k}\left(\bmod p^{2}\right)$. In particular, we confirm several conjectures of Z.W. Sun. We also pose 13 conjectures on supercongruences.
\end{abstract}

\section{INTRODUCTION}

Let $p$ be an odd prime. In 2003, Rodriguez-Villegas 11 conjectured the following congruence:

$$
\sum_{k=0}^{(p-1) / 2} \frac{\left(\begin{array}{c}
2 k \\
k
\end{array}\right)^{2}}{16^{k}} \equiv(-1)^{\frac{p-1}{2}}\left(\bmod p^{2}\right)
$$

This was later confirmed by Mortenson [7] via the Gross-Koblitz formula. See also [9] and [10, p. 204]. Recently the author's twin brother, Zhi-Wei Sun [13], obtained the congruences for $\sum_{k=0}^{p-1}\left(\begin{array}{c}2 k \\ k\end{array}\right)^{2} m^{-k}(\bmod p)$ in the cases $m=8,-16,32$ and made several conjectures for $\sum_{k=0}^{p-1}\left(\begin{array}{c}2 k \\ k\end{array}\right)^{2} m^{-k}\left(\bmod p^{2}\right)$. For example, he conjectured $(1.2)$

$$
\sum_{k=0}^{(p-1) / 2} \frac{\left(\begin{array}{c}
2 k \\
k
\end{array}\right)^{2}}{32^{k}} \equiv \begin{cases}0\left(\bmod p^{2}\right) & \text { if } 4 \mid p-3 \\
2 a-\frac{p}{2 a}\left(\bmod p^{2}\right) & \text { if } 4 \mid p-1 \text { and } p=a^{2}+b^{2} \text { with } 4 \mid a-1\end{cases}
$$

Let $\left\{P_{n}(x)\right\}$ be the Legendre polynomials given by

$$
\frac{1}{\sqrt{1-2 x t+t^{2}}}=\sum_{n=0}^{\infty} P_{n}(x) t^{n} \quad(|t|<1) .
$$

It is well known that (see [6, pp. 228-232], 4, (3.132)-(3.133)])

$$
P_{n}(x)=\frac{1}{2^{n}} \sum_{k=0}^{[n / 2]} \frac{(-1)^{k}(2 n-2 k) !}{k !(n-k) !(n-2 k) !} x^{n-2 k}=\frac{1}{2^{n} \cdot n !} \cdot \frac{d^{n}}{d x^{n}}\left(x^{2}-1\right)^{n}
$$

and $(n+1) P_{n+1}(x)=(2 n+1) x P_{n}(x)-n P_{n-1}(x)$, where $[x]$ is the greatest integer not exceeding $x$.

Received by the editors November 23, 2009 and, in revised form, May 27, 2010.

2010 Mathematics Subject Classification. Primary 11A07; Secondary 33C45, 11E25.

Key words and phrases. Legendre polynomial, congruence.

The author is supported by the Natural Sciences Foundation of China (grant No. 10971078).

(C)2010 American Mathematical Society Reverts to public domain 28 years from publication 
In the paper, by using the expansions of Legendre polynomials we obtain some congruences for $P_{\frac{p-1}{2}}(x)$ modulo $p^{2}$, where $p$ is an odd prime and $x$ is a rational $p$-integer. For example, we have

$$
\sum_{k=0}^{p-1} \frac{\left(\begin{array}{c}
2 k \\
k
\end{array}\right)^{2}}{16^{k}}\left(x^{k}-(-1)^{\frac{p-1}{2}}(1-x)^{k}\right) \equiv 0\left(\bmod p^{2}\right)
$$

and

$$
\sum_{k=0}^{\frac{p-1}{2}} \frac{\left(\begin{array}{c}
2 k \\
k
\end{array}\right)^{2}}{16^{k}}\left(x^{k}-\left(\frac{x}{p}\right) x^{-k}\right) \equiv 0(\bmod p) \quad \text { for } \quad x \neq \equiv 0(\bmod p),
$$

where $\left(\frac{x}{p}\right)$ is the Jacobi symbol. Taking $x=1$ in (1.4) we obtain (1.1) immediately, and taking $x=\frac{1}{2}$ in $(1.4)$ we deduce $(1.2)$ for $p \equiv 3(\bmod 4)$. We also determine $\sum_{k=0}^{\frac{p-1}{2}} \frac{\left(\begin{array}{c}2 k \\ k 2^{k}\end{array}\right.}{32^{2}} k(k-1) \cdots(k-r+1)\left(\bmod p^{2}\right)$ for $r \in\left\{1,2, \ldots, \frac{p-1}{2}\right\}$, $\sum_{k=0}^{[p / 3]} \frac{(3 k) !}{54^{k} \cdot k !^{3}}(\bmod p)$ and pose some conjectures on supercongruences concerning binary quadratic forms.

Throughout this paper we use $\mathbb{Z}, \mathbb{N}$ and $\mathbb{Z}_{p}$ to denote the sets of integers, positive integers and rational $p$-integers for a prime $p$, respectively.

\section{Main Results}

Lemma 2.1. For $n \in \mathbb{N}$ we have

$$
P_{n}(x)=\sum_{k=0}^{n}\left(\begin{array}{c}
n+k \\
2 k
\end{array}\right)\left(\begin{array}{c}
2 k \\
k
\end{array}\right)\left(\frac{x-1}{2}\right)^{k} .
$$

Proof. From [4, (3.135)] we have the following result due to Murphy:

$$
P_{n}(x)=\sum_{k=0}^{n}\left(\begin{array}{l}
n \\
k
\end{array}\right)\left(\begin{array}{c}
n+k \\
k
\end{array}\right)\left(\frac{x-1}{2}\right)^{k} .
$$

As $\left(\begin{array}{c}n+k \\ 2 k\end{array}\right)\left(\begin{array}{c}2 k \\ k\end{array}\right)=\left(\begin{array}{c}n+k \\ k\end{array}\right)\left(\begin{array}{c}n \\ k\end{array}\right)$, we obtain the result.

Lemma 2.2. Let $p$ be an odd prime and $k \in\{1,2, \ldots,(p-1) / 2\}$. Then

$$
\left(\begin{array}{c}
\frac{p-1}{2}+k \\
2 k
\end{array}\right) \equiv \frac{\left(\begin{array}{c}
2 k \\
k
\end{array}\right)}{(-16)^{k}}\left(1-p^{2} \sum_{i=1}^{k} \frac{1}{(2 i-1)^{2}}\right)\left(\bmod p^{4}\right) .
$$

Proof. Clearly

$$
\begin{aligned}
\left(\begin{array}{c}
\frac{p-1}{2}+k \\
2 k
\end{array}\right) & =\frac{\left(\frac{p-1}{2}+k\right)\left(\frac{p-1}{2}+k-1\right) \cdots\left(\frac{p-1}{2}-k+1\right)}{(2 k) !} \\
& =\frac{(p+2 k-1)(p+2 k-3) \cdots(p-(2 k-3))(p-(2 k-1))}{2^{2 k} \cdot(2 k) !} \\
& =\frac{\left(p^{2}-1^{2}\right)\left(p^{2}-3^{2}\right) \cdots\left(p^{2}-(2 k-1)^{2}\right)}{2^{2 k} \cdot(2 k) !} \\
& \equiv \frac{(-1)^{k} \cdot 1^{2} \cdot 3^{2} \cdots(2 k-1)^{2}}{2^{2 k} \cdot(2 k) !}\left(1-p^{2} \sum_{i=1}^{k} \frac{1}{(2 i-1)^{2}}\right)\left(\bmod p^{4}\right) .
\end{aligned}
$$


To see the result, we note that

$$
\frac{1^{2} \cdot 3^{2} \cdots(2 k-1)^{2}}{2^{2 k} \cdot(2 k) !}=\frac{(2 k) !^{2}}{(2 \cdot 4 \cdots(2 k))^{2} \cdot 2^{2 k} \cdot(2 k) !}=\frac{(2 k) !}{2^{4 k} \cdot k !^{2}}=\frac{\left(\begin{array}{c}
2 k \\
k
\end{array}\right)}{16^{k}} .
$$

Let $p$ be an odd prime, and let $\{A(n)\}$ be the Apéry numbers given by

$$
A(n)=\sum_{k=0}^{n}\left(\begin{array}{c}
n+k \\
k
\end{array}\right)^{2}\left(\begin{array}{l}
n \\
k
\end{array}\right)^{2} .
$$

It is well known that (see [1, 10] $) A\left(\frac{p-1}{2}\right) \equiv a(p)\left(\bmod p^{2}\right)$, where $a(n)$ is defined by

$$
q \prod_{n=1}^{\infty}\left(1-q^{2 n}\right)^{4}\left(1-q^{4 n}\right)^{4}=\sum_{n=1}^{\infty} a(n) q^{n} .
$$

By the fact $\left(\begin{array}{c}n+k \\ k\end{array}\right)\left(\begin{array}{c}n \\ k\end{array}\right)=\left(\begin{array}{c}n+k \\ 2 k\end{array}\right)\left(\begin{array}{c}2 k \\ k\end{array}\right)$ and Lemma 2.2 we have

$$
A\left(\frac{p-1}{2}\right)=\sum_{k=0}^{\frac{p-1}{2}}\left(\begin{array}{c}
\frac{p-1}{2}+k \\
2 k
\end{array}\right)^{2}\left(\begin{array}{c}
2 k \\
k
\end{array}\right)^{2} \equiv \sum_{k=0}^{\frac{p-1}{2}}\left(\frac{\left(\begin{array}{c}
2 k \\
k
\end{array}\right)}{(-16)^{k}}\right)^{2}\left(\begin{array}{c}
2 k \\
k
\end{array}\right)^{2}\left(\bmod p^{2}\right) .
$$

Hence

$$
a(p) \equiv A\left(\frac{p-1}{2}\right) \equiv \sum_{k=0}^{\frac{p-1}{2}} \frac{\left(\begin{array}{c}
2 k \\
k
\end{array}\right)^{4}}{4^{4 k}}\left(\bmod p^{2}\right) .
$$

Let $b(n)$ be given by $q \prod_{n=1}^{\infty}\left(1-q^{4 n}\right)^{6}=\sum_{n=1}^{\infty} b(n) q^{n}$. Then Mortenson [8] proved the following conjecture of Rodriguez-Villegas:

$$
\sum_{k=1}^{\frac{p-1}{2}} \frac{\left(\begin{array}{c}
2 k \\
k
\end{array}\right)^{3}}{4^{3 k}} \equiv b(p)\left(\bmod p^{2}\right)
$$

Theorem 2.1. Let $p$ be an odd prime and let $x$ be a variable. Then

$$
\sum_{k=0}^{p-1} \frac{\left(\begin{array}{c}
2 k \\
k
\end{array}\right)^{2}}{16^{k}}\left(x^{k}-(-1)^{\frac{p-1}{2}}(1-x)^{k}\right) \equiv \sum_{k=0}^{\frac{p-1}{2}} \frac{\left(\begin{array}{c}
2 k \\
k
\end{array}\right)^{2}}{16^{k}}\left(x^{k}-(-1)^{\frac{p-1}{2}}(1-x)^{k}\right) \equiv 0\left(\bmod p^{2}\right) \text {. }
$$

Proof. For a variable $t$, by Lemmas 2.1 and 2.2 we have

$$
P_{\frac{p-1}{2}}(t)=\sum_{k=0}^{\frac{p-1}{2}}\left(\begin{array}{c}
\frac{p-1}{2}+k \\
2 k
\end{array}\right)\left(\begin{array}{c}
2 k \\
k
\end{array}\right)\left(\frac{t-1}{2}\right)^{k} \equiv \sum_{k=0}^{\frac{p-1}{2}} \frac{\left(\begin{array}{c}
2 k \\
k
\end{array}\right)^{2}}{16^{k}}\left(\frac{1-t}{2}\right)^{k}\left(\bmod p^{2}\right) .
$$

It is known that (see [6]) $P_{n}(t)=(-1)^{n} P_{n}(-t)$. Thus, by $(2.4)$,

$$
\sum_{k=0}^{\frac{p-1}{2}} \frac{\left(\begin{array}{c}
2 k \\
k
\end{array}\right)^{2}}{16^{k}}\left(\frac{1-t}{2}\right)^{k} \equiv(-1)^{\frac{p-1}{2}} \sum_{k=0}^{\frac{p-1}{2}} \frac{\left(\begin{array}{c}
2 k \\
k
\end{array}\right)^{2}}{16^{k}}\left(\frac{1+t}{2}\right)^{k}\left(\bmod p^{2}\right) .
$$

Now taking $t=1-2 x$ in the congruence we deduce that

$$
\sum_{k=0}^{\frac{p-1}{2}} \frac{\left(\begin{array}{c}
2 k \\
k
\end{array}\right)^{2}}{16^{k}}\left(x^{k}-(-1)^{\frac{p-1}{2}}(1-x)^{k}\right) \equiv 0\left(\bmod p^{2}\right) .
$$

To complete the proof, we note that for $k \in\left\{\frac{p+1}{2}, \frac{p+3}{2}, \ldots, p-1\right\},\left(\begin{array}{c}2 k \\ k\end{array}\right)=$ $2 k(2 k-1) \cdots(k+1) / k ! \equiv 0(\bmod p)$. 
Theorem 2.2. Let $p$ be an odd prime. Then

$$
\sum_{k=0}^{\frac{p-1}{2}} \frac{\left(\begin{array}{c}
2 k \\
k
\end{array}\right)^{2}}{32^{k}} \equiv \begin{cases}0\left(\bmod p^{2}\right) & \text { if } 4 \mid p-3, \\
2 a-\frac{p}{2 a}\left(\bmod p^{2}\right) & \text { if } 4 \mid p-1 \text { and } p=a^{2}+b^{2} \text { with } 4 \mid a-1 .\end{cases}
$$

Proof. When $p \equiv 3(\bmod 4)$, taking $x=\frac{1}{2}$ in Theorem 2.1 we obtain the result. Now suppose $p \equiv 1(\bmod 4)$ and so $p=a^{2}+b^{2}$ with $a, b \in \mathbb{Z}$ and $a \equiv 1(\bmod 4)$. It is well known that $([6])$

$$
P_{2 n+1}(0)=0 \quad \text { and } \quad P_{2 n}(0)=\frac{(-1)^{n}}{2^{2 n}}\left(\begin{array}{c}
2 n \\
n
\end{array}\right) .
$$

Thus, by (2.4) and (2.5) we have

$$
\sum_{k=0}^{\frac{p-1}{2}} \frac{\left(\begin{array}{c}
2 k \\
k
\end{array}\right)^{2}}{32^{k}} \equiv P_{\frac{p-1}{2}}(0)=\frac{(-1)^{\frac{p-1}{4}}}{2^{\frac{p-1}{2}}}\left(\begin{array}{c}
\frac{p-1}{2} \\
\frac{p-1}{4}
\end{array}\right)\left(\bmod p^{2}\right)
$$

According to the result due to Chowla, Dwork and Evans (see [2] or [3]), we have

$$
\left(\begin{array}{c}
\frac{p-1}{2} \\
\frac{p-1}{4}
\end{array}\right) \equiv \frac{2^{p-1}+1}{2}\left(2 a-\frac{p}{2 a}\right)\left(\bmod p^{2}\right) .
$$

Set $q=\left(2^{\frac{p-1}{2}}-(-1)^{\frac{p-1}{4}}\right) / p$. Then $2^{p-1} \equiv 1+2(-1)^{\frac{p-1}{4}} q p\left(\bmod p^{2}\right)$. Thus

$$
\frac{2^{p-1}+1}{2 \cdot 2^{\frac{p-1}{2}}} \equiv \frac{2+2(-1)^{\frac{p-1}{4}} q p}{2\left((-1)^{\frac{p-1}{4}}+q p\right)}=(-1)^{\frac{p-1}{4}}\left(\bmod p^{2}\right) \text {. }
$$

Hence

$$
\sum_{k=0}^{\frac{p-1}{2}} \frac{\left(\begin{array}{c}
2 k \\
k
\end{array}\right)^{2}}{32^{k}} \equiv \frac{(-1)^{\frac{p-1}{4}}}{2^{\frac{p-1}{2}}}\left(\begin{array}{c}
\frac{p-1}{2} \\
\frac{p-1}{4}
\end{array}\right) \equiv \frac{(-1)^{\frac{p-1}{4}}}{2^{\frac{p-1}{2}}} \cdot \frac{2^{p-1}+1}{2}\left(2 a-\frac{p}{2 a}\right) \equiv 2 a-\frac{p}{2 a}\left(\bmod p^{2}\right) .
$$

The proof is now complete.

Remark 2.1. Theorem 2.2 was conjectured by Zhi-Wei Sun ([13]), and the congruence for $\sum_{k=0}^{\frac{p-1}{2}} \frac{\left(\begin{array}{c}2 k \\ k\end{array}\right)^{2}}{32^{k}}(\bmod p)$ was also proved by Zhi-Wei Sun in [13].

Theorem 2.3. Let $p$ be an odd prime and $r \in\{1,2, \ldots,(p-1) / 2\}$. Then

$$
\begin{aligned}
& \sum_{k=0}^{\frac{p-1}{2}} \frac{\left(\begin{array}{c}
2 k \\
k
\end{array}\right)^{2}}{32^{k}} k(k-1) \cdots(k-r+1) \\
& \equiv \begin{cases}0\left(\bmod p^{2}\right) & \text { if } 4 \mid(p+1-2 r), \\
(-1)^{\frac{p-1+2 r}{4}} 2^{-\frac{p-1}{2}} \frac{\left(\frac{p-1}{2}+r\right) !}{\frac{p-1-2 r}{4} ! \frac{p-1+2 r}{4} !}\left(\bmod p^{2}\right) & \text { if } 4 \mid(p-1-2 r) .\end{cases}
\end{aligned}
$$

Proof. By (2.4) we have

$$
\begin{aligned}
\frac{d^{r} P_{\frac{p-1}{2}}(t)}{d t^{r}} & \equiv \sum_{k=0}^{\frac{p-1}{2}} \frac{\left(\begin{array}{c}
2 k \\
k
\end{array}\right)^{2}}{(-32)^{k}} \cdot \frac{d^{r}(t-1)^{k}}{d t^{r}} \\
& =\sum_{k=0}^{\frac{p-1}{2}} \frac{\left(\begin{array}{c}
2 k \\
k
\end{array}\right)^{2}}{(-32)^{k}} k(k-1) \cdots(k-r+1)(t-1)^{k-r}\left(\bmod p^{2}\right) .
\end{aligned}
$$


Hence

$$
\left.\frac{d^{r} P_{\frac{p-1}{2}}(t)}{d t^{r}}\right|_{t=0}=(-1)^{r} \sum_{k=0}^{\frac{p-1}{2}} \frac{\left(\begin{array}{c}
2 k \\
k
\end{array}\right)^{2}}{32^{k}} k(k-1) \cdots(k-r+1) .
$$

By (1.3) we have

$$
\begin{aligned}
\frac{d^{r}}{d t^{r}} P_{\frac{p-1}{2}}(t)= & \frac{1}{2^{(p-1) / 2}} \cdot \frac{d^{r}}{d t^{r}} \sum_{m=0}^{\left.\frac{p-1}{4}\right]} \frac{(-1)^{m}(p-1-2 m) !}{m !\left(\frac{p-1}{2}-m\right) !\left(\frac{p-1}{2}-2 m\right) !} t^{\frac{p-1}{2}-2 m} \\
= & \frac{1}{2^{(p-1) / 2}} \sum_{m=0}^{\left[\frac{p-1-2 r}{4}\right]} \frac{(-1)^{m}(p-1-2 m) !}{m !\left(\frac{p-1}{2}-m\right) !\left(\frac{p-1}{2}-2 m\right) !} \\
& \times\left(\frac{p-1}{2}-2 m\right)\left(\frac{p-1}{2}-2 m-1\right) \cdots\left(\frac{p-1}{2}-2 m-r+1\right) t^{\frac{p-1}{2}-2 m-r} .
\end{aligned}
$$

Thus,

$$
\left.\frac{d^{r} P_{\frac{p-1}{2}}(t)}{d t^{r}}\right|_{t=0}= \begin{cases}0 & \text { if } r \not \equiv \frac{p-1}{2}(\bmod 2), \\ \frac{(-1)^{m}(p-1-2 m) !}{2^{(p-1) / 2} \cdot m !\left(\frac{p-1}{2}-m\right) !} & \text { if } r=\frac{p-1}{2}-2 m .\end{cases}
$$

Now combining all the above we obtain the result.

Corollary 2.1. Let $p$ be an odd prime. Then

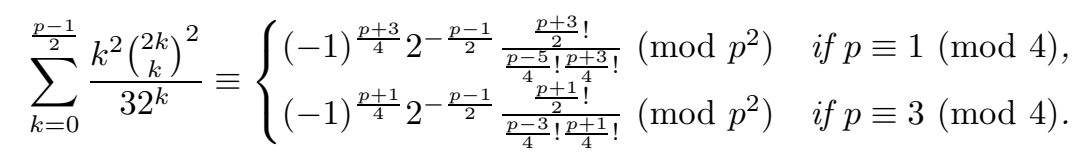

Proof. By Theorem 2.3 we have

$$
\sum_{k=0}^{\frac{p-1}{2}} \frac{k\left(\begin{array}{c}
2 k \\
k
\end{array}\right)^{2}}{32^{k}} \equiv \begin{cases}0\left(\bmod p^{2}\right) & \text { if } p \equiv 1(\bmod 4) \\
(-1)^{\frac{p+1}{4}} 2^{-\frac{p-1}{2}} \frac{\frac{p+1}{2} !}{\frac{p-3}{4} ! \frac{p+1}{4} !}\left(\bmod p^{2}\right) & \text { if } p \equiv 3(\bmod 4)\end{cases}
$$

and

$$
\sum_{k=0}^{\frac{p-1}{2}} \frac{k(k-1)\left(\begin{array}{c}
2 k \\
k
\end{array}\right)^{2}}{32^{k}} \equiv \begin{cases}(-1)^{\frac{p+3}{4}} 2^{-\frac{p-1}{2}} \frac{p-3 !}{\frac{p-5}{4} ! \frac{p+3}{4} !}\left(\bmod p^{2}\right) & \text { if } p \equiv 1(\bmod 4) \\
0\left(\bmod p^{2}\right) & \text { if } p \equiv 3(\bmod 4)\end{cases}
$$

Observe that $k^{2}=k(k-1)+k$. From the above we deduce the result.

Lemma 2.3. Let $p$ be a prime greater than 3 and let $t$ be a variable. Then

$$
P_{\left[\frac{p}{3}\right]}(t) \equiv \sum_{k=0}^{[p / 3]} \frac{(3 k) !}{k !^{3}}\left(\frac{1-t}{54}\right)^{k}(\bmod p) .
$$


Proof. Suppose $r=1$ or 2 according to whether $3 \mid p-1$ or $3 \mid p-2$. Then clearly

$$
\begin{aligned}
\left(\begin{array}{c}
\frac{p-r}{3}+k \\
2 k
\end{array}\right) & =\frac{\left(\frac{p-r}{3}+k\right)\left(\frac{p-r}{3}+k-1\right) \cdots\left(\frac{p-r}{3}-k+1\right)}{(2 k) !} \\
& =\frac{(p+3 k-r)(p+3 k-r-3) \cdots(p-(3 k+r-3))}{3^{2 k} \cdot(2 k) !} \\
& \equiv(-1)^{k} \frac{(3 k-r)(3 k-r-3) \cdots(3-r) \cdot r(r+3) \cdots(3 k+r-3)}{3^{2 k} \cdot(2 k) !} \\
& =\frac{(-1)^{k} \cdot(3 k) !}{3 \cdot 6 \cdots 3 k \cdot 3^{2 k} \cdot(2 k) !}=\frac{(-1)^{k} \cdot(3 k) !}{3^{k} \cdot k ! \cdot 3^{2 k} \cdot(2 k) !}(\bmod p) .
\end{aligned}
$$

Hence, by Lemma 2.1 we have

$$
\begin{aligned}
P_{\left[\frac{p}{3}\right]}(t) & =\sum_{k=0}^{[p / 3]}\left(\begin{array}{c}
{\left[\frac{p}{3}\right]+k} \\
2 k
\end{array}\right)\left(\begin{array}{c}
2 k \\
k
\end{array}\right)\left(\frac{t-1}{2}\right)^{k} \equiv \sum_{k=0}^{[p / 3]} \frac{(-1)^{k} \cdot(3 k) !}{3^{3 k} \cdot k !(2 k) !} \cdot \frac{(2 k) !}{k !^{2}}\left(\frac{t-1}{2}\right)^{k} \\
& =\sum_{k=0}^{[p / 3]} \frac{(3 k) !}{27^{k} \cdot k !^{3}}\left(\frac{1-t}{2}\right)^{k}(\bmod p) .
\end{aligned}
$$

This proves the lemma.

Theorem 2.4. Let $p$ be a prime greater than 3 and let $x$ be a variable. Then

$$
\sum_{k=0}^{[p / 3]} \frac{(3 k) !}{27^{k} \cdot k !^{3}}\left(x^{k}-(-1)^{\left[\frac{p}{3}\right]}(1-x)^{k}\right) \equiv 0(\bmod p) .
$$

Proof. As $P_{n}(t)=(-1)^{n} P_{n}(-t)$, using Lemma 2.3 we deduce that

$$
\sum_{k=0}^{[p / 3]} \frac{(3 k) !}{27^{k} \cdot k !^{3}}\left(\left(\frac{1-t}{2}\right)^{k}-(-1)^{[p / 3]}\left(\frac{1+t}{2}\right)^{k}\right) \equiv 0(\bmod p) .
$$

Now putting $t=1-2 x$ in the congruence we obtain the result.

Corollary 2.2. Let $p$ be a prime greater than 3 . Then

$$
\sum_{k=0}^{[p / 3]} \frac{(3 k) !}{27^{k} \cdot k !^{3}} \equiv\left(\frac{p}{3}\right)(\bmod p) \text {. }
$$

Proof. Taking $x=1$ in Theorem 2.4 and noting that $(-1)^{[p / 3]}=\left(\frac{p}{3}\right)$ we deduce the result.

Remark 2.2. By [8] or [10, p. 204] we have the stronger supercongruence $\sum_{k=0}^{p-1} \frac{(3 k) !}{27^{k} \cdot k !^{3}} \equiv\left(\frac{p}{3}\right)\left(\bmod p^{2}\right)$.

Lemma 2.4. Let $p$ be an odd prime and $k \in\left\{1,2, \ldots, \frac{p-1}{2}\right\}$. Then

$$
\left(\begin{array}{c}
(p-1) / 2 \\
k
\end{array}\right) \equiv \frac{1}{(-4)^{k}}\left(\begin{array}{c}
2 k \\
k
\end{array}\right)\left(1-p \sum_{i=1}^{k} \frac{1}{2 i-1}\right)\left(\bmod p^{2}\right) .
$$


Proof. It is clear that

$$
\begin{aligned}
\left(\begin{array}{c}
\frac{p-1}{2} \\
k
\end{array}\right) & =\frac{\frac{p-1}{2}\left(\frac{p-1}{2}-1\right) \cdots\left(\frac{p-1}{2}-k+1\right)}{k !}=\frac{(p-1)(p-3) \cdots(p-(2 k-1))}{2^{k} \cdot k !} \\
& \equiv \frac{(-1)(-3) \cdots(-(2 k-1))}{2^{k} \cdot k !}\left(1-p \sum_{i=1}^{k} \frac{1}{2 i-1}\right) \\
& =\frac{(-1)^{k} \cdot(2 k) !}{\left(2^{k} \cdot k !\right)^{2}}\left(1-p \sum_{i=1}^{k} \frac{1}{2 i-1}\right)\left(\bmod p^{2}\right) .
\end{aligned}
$$

This yields the result.

Theorem 2.5. Let $p$ be a prime greater than 5 . Then

$$
\sum_{k=0}^{[p / 3]} \frac{(3 k) !}{54^{k} \cdot k !^{3}} \equiv \begin{cases}0(\bmod p) & \text { if } 6 \mid p-5, \\ 2 A(\bmod p) & \text { if } 6 \mid p-1 \text { and } p=A^{2}+3 B^{2} \text { with } 3 \mid A-1\end{cases}
$$

and

$$
\sum_{k=0}^{[p / 3]} \frac{k \cdot(3 k) !}{54^{k} \cdot k !^{3}} \equiv \begin{cases}0(\bmod p) & \text { if } 6 \mid p-1 \\
\frac{1}{3}(-1)^{\frac{p+1}{6}} 2^{-\frac{p+1}{3}}\left(\begin{array}{l}
(p+1) / 3 \\
(p+1) / 6
\end{array}\right)(\bmod p) & \text { if } 6 \mid p-5 .\end{cases}
$$

Proof. Taking $t=0$ in Lemma 2.3 and applying (2.5) and Lemma 2.4 we deduce that

$$
\sum_{k=0}^{[p / 3]} \frac{(3 k) !}{54^{k} \cdot k !^{3}} \equiv \begin{cases}0(\bmod p) & \text { if } p \equiv 5(\bmod 6) \\
\frac{(-1)^{(p-1) / 6}}{2^{(p-1) / 3}}\left(\begin{array}{c}
(p-1) / 3 \\
(p-1) / 6
\end{array}\right) \equiv\left(\begin{array}{l}
(p-1) / 2 \\
(p-1) / 6
\end{array}\right)(\bmod p) & \text { if } p \equiv 1(\bmod 6) .\end{cases}
$$

Now suppose $p \equiv 1(\bmod 6)$, and so $p=A^{2}+3 B^{2}$ with $A, B \in \mathbb{Z}$ and $A \equiv 1(\bmod 3)$. By [2, Theorem 9.4.4] we have $\left(\begin{array}{c}(p-1) / 2 \\ (p-1) / 6\end{array}\right) \equiv 2 A(\bmod p)$. Thus the first part follows.

By Lemma 2.3 we have

$$
\frac{d}{d t} P_{\left[\frac{p}{3}\right]}(t) \equiv-\sum_{k=0}^{[p / 3]} \frac{(3 k) !}{54^{k} \cdot k !^{3}} \cdot k(1-t)^{k-1}(\bmod p) .
$$

Thus, $\left.\frac{d}{d t} P_{\left[\frac{p}{3}\right]}(t)\right|_{t=0} \equiv-\sum_{k=0}^{[p / 3]} \frac{k \cdot(3 k) !}{54^{k} \cdot k !^{3}}(\bmod p)$. From (1.3) we know that

$$
\left.\frac{d}{d t} P_{\left[\frac{p}{3}\right]}(t)\right|_{t=0}= \begin{cases}0 & \text { if } p \equiv 1(\bmod 6) \\ 2^{-\frac{p-2}{3}} \cdot(-1)^{\frac{p-5}{6}} \frac{p+3}{\frac{p-5}{6} ! \frac{p+1}{6} !} & \text { if } p \equiv 5(\bmod 6) .\end{cases}
$$

Thus the second part is true.

Lemma 2.5. Let $p$ be an odd prime and $k \in\left\{1,2, \ldots, \frac{p-1}{2}\right\}$. Then

$$
\frac{(-1)^{k}\left(\begin{array}{c}
(p-1) / 2+k \\
k
\end{array}\right)}{\left(\begin{array}{c}
(p-1) / 2 \\
k
\end{array}\right)} \equiv 1+2 p \sum_{i=1}^{k} \frac{1}{2 i-1} \equiv 3-2(-4)^{k} \frac{\left(\begin{array}{c}
(p-1) / 2 \\
k
\end{array}\right)}{\left(\begin{array}{c}
2 k \\
k
\end{array}\right)}\left(\bmod p^{2}\right) .
$$


Proof. It is clear that

$$
\begin{aligned}
\frac{(-1)^{k}\left(\begin{array}{c}
(p-1) / 2+k \\
k
\end{array}\left(\begin{array}{c}
(p-1) / 2 \\
k
\end{array}\right)\right.}{} & =\frac{\left(\frac{p-1}{2}+k\right)\left(\frac{p-1}{2}+k-1\right) \cdots\left(\frac{p-1}{2}+1\right)}{(-1)^{k} \frac{p-1}{2}\left(\frac{p-1}{2}-1\right) \cdots\left(\frac{p-1}{2}-k+1\right)} \\
& =\frac{(p+2 k-1)(p+2 k-3) \cdots(p+1)}{(-1)^{k}(p-1)(p-3) \cdots(p-(2 k-1))} \\
& \equiv \frac{1 \cdot 3 \cdots(2 k-1)\left(1+p \sum_{i=1}^{k} \frac{1}{2 i-1}\right)}{1 \cdot 3 \cdots(2 k-1)\left(1-p \sum_{i=1}^{k} \frac{1}{2 i-1}\right)} \\
& \equiv\left(1+p \sum_{i=1}^{k} \frac{1}{2 i-1}\right)^{2} \equiv 1+2 p \sum_{i=1}^{k} \frac{1}{2 i-1}\left(\bmod p^{2}\right) .
\end{aligned}
$$

This together with Lemma 2.4 yields the result.

Theorem 2.6. Let $p$ be an odd prime, $x \in \mathbb{Z}_{p}$ and $x \not \equiv-1(\bmod p)$. Then

$$
P_{\frac{p-1}{2}}(x) \equiv\left(\frac{2(x+1)}{p}\right) P_{\frac{p-1}{2}}\left(\frac{3-x}{1+x}\right)(\bmod p) .
$$

Proof. It is known that (see [4, (3.134)])

$$
P_{n}(x)=\sum_{k=0}^{n}\left(\begin{array}{l}
n \\
k
\end{array}\right)^{2}\left(\frac{x+1}{2}\right)^{n-k}\left(\frac{x-1}{2}\right)^{k} .
$$

Thus, using Lemma 2.5 and (2.1) we see that

$$
\begin{aligned}
P_{\frac{p-1}{2}}(x) & =\left(\frac{x+1}{2}\right)^{\frac{p-1}{2}} \sum_{k=0}^{\frac{p-1}{2}}\left(\begin{array}{c}
\frac{p-1}{2} \\
k
\end{array}\right)^{2}\left(\frac{x-1}{x+1}\right)^{k} \\
& \equiv\left(\frac{2(x+1)}{p}\right) \sum_{k=0}^{\frac{p-1}{2}}\left(\begin{array}{c}
\frac{p-1}{2} \\
k
\end{array}\right)\left(\begin{array}{c}
\frac{p-1}{2}+k \\
k
\end{array}\right)(-1)^{k}\left(\frac{x-1}{x+1}\right)^{k} \\
& =\left(\frac{2(x+1)}{p}\right) P_{\frac{p-1}{2}}\left(1+2 \cdot \frac{1-x}{1+x}\right)=\left(\frac{2(x+1)}{p}\right) P_{\frac{p-1}{2}}\left(\frac{3-x}{1+x}\right)(\bmod p) .
\end{aligned}
$$

This proves the theorem.

Corollary 2.3. Let $p$ be a prime of the form $4 k+3$. Then $P_{\frac{p-1}{2}}(3) \equiv 0(\bmod p)$.

Proof. By Theorem 2.6 and $(2.5)$ we have $P_{\frac{p-1}{2}}(3) \equiv\left(\frac{2}{p}\right) P_{\frac{p-1}{2}}(0)=0(\bmod p)$.

Theorem 2.7. Let $p$ be an odd prime, $x \in \mathbb{Z}_{p}$ and $x \not \equiv 0(\bmod p)$. Then

$$
\sum_{k=0}^{\frac{p-1}{2}} \frac{\left(\begin{array}{c}
2 k \\
k
\end{array}\right)^{2}}{16^{k}}\left(x^{k}-\left(\frac{x}{p}\right) x^{-k}\right) \equiv 0(\bmod p) .
$$

Proof. Clearly the result is true for $x \equiv 1(\bmod p)$. Now assume $x \not \equiv 1(\bmod p)$. As $P_{n}(t)=(-1)^{n} P_{n}(-t)$ (see [6]), using Theorem 2.6 we see that for $t \in \mathbb{Z}_{p}$ with $t \not \equiv \pm 1(\bmod p)$

$$
\left(\frac{2(t+1)}{p}\right) P_{\frac{p-1}{2}}\left(\frac{3-t}{1+t}\right) \equiv(-1)^{\frac{p-1}{2}}\left(\frac{2(-t+1)}{p}\right) P_{\frac{p-1}{2}}\left(\frac{3+t}{1-t}\right)(\bmod p) .
$$


Thus,

$$
P_{\frac{p-1}{2}}\left(\frac{3-t}{1+t}\right) \equiv\left(\frac{t^{2}-1}{p}\right) P_{\frac{p-1}{2}}\left(\frac{3+t}{1-t}\right)(\bmod p) .
$$

Now applying (2.4) we deduce that

$$
\sum_{k=0}^{\frac{p-1}{2}} \frac{\left(\begin{array}{c}
2 k \\
k
\end{array}\right)^{2}}{16^{k}}\left(\frac{1-\frac{3-t}{1+t}}{2}\right)^{k} \equiv\left(\frac{t^{2}-1}{p}\right) \sum_{k=0}^{\frac{p-1}{2}} \frac{\left(\begin{array}{c}
2 k \\
k
\end{array}\right)^{2}}{16^{k}}\left(\frac{1-\frac{3+t}{1-t}}{2}\right)^{k}(\bmod p)
$$

and so

$$
\sum_{k=0}^{\frac{p-1}{2}} \frac{\left(\begin{array}{c}
2 k \\
k
\end{array}\right)^{2}}{16^{k}}\left(\left(\frac{t-1}{t+1}\right)^{k}-\left(\frac{(t-1) /(t+1)}{p}\right)\left(\frac{t+1}{t-1}\right)^{k}\right) \equiv 0(\bmod p) .
$$

Set $t=(1+x) /(1-x)$. Then $t \not \equiv \pm 1(\bmod p)$ and $x=(t-1) /(t+1)$. Hence the result follows.

Let $p$ be an odd prime, and let $x \in \mathbb{Z}_{p}$ with $x \not \equiv 0,1(\bmod p)$. By Theorems 2.1 and 2.7 we have

$$
\sum_{k=0}^{(p-1) / 2} \frac{\left(\begin{array}{c}
2 k \\
k
\end{array}\right)^{2}}{(16 x)^{k}} \equiv\left(\frac{x(x-1)}{p}\right) \sum_{k=0}^{(p-1) / 2} \frac{\left(\begin{array}{c}
2 k \\
k
\end{array}\right)^{2}}{(16(1-x))^{k}}(\bmod p) .
$$

Theorem 2.8. Let $p$ be an odd prime, $x \in \mathbb{Z}_{p}$ and $x \not \equiv 0(\bmod p)$. Then

$$
\sum_{k=0}^{\frac{p-1}{2}}\left(\begin{array}{c}
2 k \\
k
\end{array}\right)^{2}\left(\frac{x}{4}\right)^{2 k} \equiv\left(\frac{-x}{p}\right) P_{\frac{p-1}{2}}\left(\frac{x+x^{-1}}{2}\right)(\bmod p) .
$$

Proof. From [4, (3.138)] we have the following result due to Kelisky:

$$
\sum_{k=0}^{n}\left(\begin{array}{c}
2 n-2 k \\
n-k
\end{array}\right)\left(\begin{array}{c}
2 k \\
k
\end{array}\right) x^{2 k}=2^{2 n} x^{n} P_{n}\left(\frac{x+x^{-1}}{2}\right) .
$$

Taking $n=(p-1) / 2$ in $(2.8)$ we have

$$
\sum_{k=0}^{\frac{p-1}{2}}\left(\begin{array}{c}
p-1-2 k \\
\frac{p-1}{2}-k
\end{array}\right)\left(\begin{array}{c}
2 k \\
k
\end{array}\right) x^{2 k}=2^{p-1} x^{\frac{p-1}{2}} P_{\frac{p-1}{2}}\left(\frac{x+x^{-1}}{2}\right) \equiv\left(\frac{x}{p}\right) P_{\frac{p-1}{2}}\left(\frac{x+x^{-1}}{2}\right)(\bmod p) .
$$

To see the result, using Lemma 2.2 we note that for $0 \leq k \leq \frac{p-1}{2}$,

$$
\begin{aligned}
\left(\begin{array}{c}
p-1-2 k \\
\frac{p-1}{2}-k
\end{array}\right) & =\frac{(p-1-2 k)(p-2-2 k) \cdots\left(p-\left(\frac{p-1}{2}+k\right)\right)}{\left(\frac{p-1}{2}-k\right) !} \\
& \equiv(-1)^{\frac{p-1}{2}-k} \frac{(2 k+1)(2 k+2) \cdots\left(\frac{p-1}{2}+k\right)}{\left(\frac{p-1}{2}-k\right) !} \\
& =(-1)^{\frac{p-1}{2}-k}\left(\begin{array}{c}
\frac{p-1}{2}+k \\
2 k
\end{array}\right) \equiv(-1)^{\frac{p-1}{2}} \frac{\left(\begin{array}{c}
2 k \\
k
\end{array}\right)}{16^{k}}(\bmod p) .
\end{aligned}
$$


Theorem 2.9. Let $p$ be a prime of the form $4 k+1$ and $p=a^{2}+b^{2}$ with $a, b \in \mathbb{Z}$ and $a \equiv 1(\bmod 4)$. Then

$$
\sum_{k=0}^{\frac{p-1}{2}} \frac{\left(\begin{array}{c}
2 k \\
k
\end{array}\right)^{2}}{8^{k}} \equiv \sum_{k=0}^{\frac{p-1}{2}} \frac{\left(\begin{array}{c}
2 k \\
k
\end{array}\right)^{2}}{(-16)^{k}} \equiv P_{\frac{p-1}{2}}(3) \equiv(-1)^{\frac{p-1}{4}}\left(2 a-\frac{p}{2 a}\right)\left(\bmod p^{2}\right) .
$$

Proof. By Theorem 2.1 and (2.4) we have $\sum_{k=0}^{\frac{p-1}{2}}\left(\begin{array}{c}2 k \\ k\end{array}\right)^{2} 8^{-k} \equiv \sum_{k=0}^{\frac{p-1}{2}}\left(\begin{array}{c}2 k \\ k\end{array}\right)^{2}(-16)^{-k} \equiv$ $P_{\frac{p-1}{2}}(3)\left(\bmod p^{2}\right)$. From Theorem 2.6, (2.5) and Gauss' congruence $\left(\begin{array}{c}(p-1) / 2 \\ (p-1) / 4\end{array}\right) \equiv 2 a$ $(\bmod p)($ see $[2])$ we have

$$
P_{\frac{p-1}{2}}(3) \equiv\left(\frac{2}{p}\right) P_{\frac{p-1}{2}}(0)=\left(\frac{2}{p}\right) \frac{(-1)^{(p-1) / 4}}{2^{(p-1) / 2}}\left(\begin{array}{c}
(p-1) / 2 \\
(p-1) / 4
\end{array}\right) \equiv(-1)^{\frac{p-1}{4}} \cdot 2 a(\bmod p) .
$$

Write $P_{\frac{p-1}{2}}(3)=(-1)^{\frac{p-1}{4}} \cdot 2 a+q p$. Then $P_{\frac{p-1}{2}}(3)^{2} \equiv 4 a^{2}+(-1)^{\frac{p-1}{4}} \cdot 4 a q p\left(\bmod p^{2}\right)$. By a result due to Van Hamme [15, we have $\left(\sum_{k=0}^{\frac{p-1}{2}}\left(\frac{\frac{p-1}{2}}{k}\right)\left(\frac{p-1}{2}+k\right)\right)^{2} \equiv 4 a^{2}-$ $2 p\left(\bmod p^{2}\right)$. This together with $(2.1)$ yields $P_{\frac{p-1}{2}}(3)^{2} \equiv 4 a^{2}-2 p\left(\bmod p^{2}\right)$. Hence $(-1)^{\frac{p-1}{4}} \cdot 4 a q \equiv-2(\bmod p)$, and so $P_{\frac{p-1}{2}}(3) \equiv(-1)^{\frac{p-1}{4}} \cdot 2 a-\frac{p}{2(-1)^{(p-1) / 4} a}\left(\bmod p^{2}\right)$. Now combining all the above we obtain the result.

Remark 2.3. For a prime $p=4 k+1=a^{2}+b^{2}$ with $a \equiv 1(\bmod 4)$, the congruence $\sum_{k=0}^{(p-1) / 2}\left(\begin{array}{c}2 k \\ k\end{array}\right)^{2} 8^{-k} \equiv \sum_{k=0}^{(p-1) / 2}\left(\begin{array}{c}2 k \\ k\end{array}\right)^{2}(-16)^{-k} \equiv(-1)^{\frac{p-1}{4}} \cdot 2 a(\bmod p)$ was proved by Zhi-Wei Sun in [13], and he also conjectured

$$
\begin{aligned}
\sum_{k=0}^{(p-1) / 2}\left(\begin{array}{c}
2 k \\
k
\end{array}\right)^{2} 8^{-k} & \equiv \sum_{k=0}^{(p-1) / 2}\left(\begin{array}{c}
2 k \\
k
\end{array}\right)^{2}(-16)^{-k} \\
& \equiv(-1)^{\frac{p-1}{4}}\left(2 a-\frac{p}{2 a}\right)\left(\bmod p^{2}\right) .
\end{aligned}
$$

Theorem 2.10. Let $p$ be a prime of the form $4 k+1$, and so $p=a^{2}+b^{2}$ with $a, b \in \mathbb{Z}$ and $a \equiv 1(\bmod 4)$. Then

$$
\sum_{k=0}^{\frac{p-1}{4}} \frac{\left(\begin{array}{l}
4 k \\
2 k
\end{array}\right)^{2}}{16^{2 k}} \equiv \frac{1}{2}+(-1)^{\frac{p-1}{4}} a-(-1)^{\frac{p-1}{4}} \frac{p}{4 a}\left(\bmod p^{2}\right) .
$$

Proof. Since $\sum_{k=0}^{\frac{p-1}{2}}\left(\begin{array}{c}2 k \\ k\end{array}\right)^{2} 16^{-k}+\sum_{k=0}^{\frac{p-1}{2}}\left(\begin{array}{c}2 k \\ k\end{array}\right)^{2}(-16)^{-k}=2 \sum_{k=0}^{\frac{p-1}{4}}\left(\begin{array}{c}4 k \\ 2 k\end{array}\right)^{2} 16^{-2 k}$, by (1.1) and Theorem 2.9 we deduce the result.

For a prime $p>3$ and $A, B, C \in \mathbb{Z}_{p}$, and let $\# E_{p}\left(y^{2}=x^{3}+A x^{2}+B x+C\right)$ be the number of points on the curve $E_{p}: y^{2}=x^{3}+A x^{2}+B x+C$ over the field $\mathbb{F}_{p}$ of $p$ elements.

Lemma $2.6([5])$. Let $p>3$ be a prime and $\lambda \in \mathbb{Z}_{p}$ with $\lambda \not \equiv 0,1(\bmod p)$. Then $p+1-\# E_{p}\left(y^{2}=x(x-1)(x-\lambda)\right) \equiv(-1)^{\frac{p-1}{2}} \sum_{k=0}^{\frac{p-1}{2}}\left(\begin{array}{c}\frac{p-1}{2}+k \\ k\end{array}\right)\left(\begin{array}{c}\frac{p-1}{2} \\ k\end{array}\right)(-\lambda)^{k}(\bmod p)$.

Theorem 2.11. Let $p>3$ be a prime and $t \in \mathbb{Z}_{p}$. Then

$$
P_{\frac{p-1}{2}}(t) \equiv \sum_{k=0}^{\frac{p-1}{2}}\left(\begin{array}{c}
2 k \\
k
\end{array}\right)^{2}\left(\frac{1-t}{32}\right)^{k} \equiv-\left(\frac{-6}{p}\right) \sum_{x=0}^{p-1}\left(\frac{x^{3}-3\left(t^{2}+3\right) x+2 t\left(t^{2}-9\right)}{p}\right)(\bmod p) .
$$


Proof. For $t \equiv \pm 1(\bmod p)$ we have

$$
\begin{aligned}
& \sum_{x=0}^{p-1}\left(\frac{x^{3}-3\left(t^{2}+3\right) x+2 t\left(t^{2}-9\right)}{p}\right) \\
& =\sum_{x=0}^{p-1}\left(\frac{x^{3}-12 x \mp 16}{p}\right)=\sum_{x=0}^{p-1}\left(\frac{(2 x)^{3}-12(2 x) \mp 16}{p}\right)=\left(\frac{2}{p}\right) \sum_{x=0}^{p-1}\left(\frac{x^{3}-3 x \mp 2}{p}\right) \\
& =\left(\frac{2}{p}\right) \sum_{x=0}^{p-1}\left(\frac{(x \pm 1)^{2}(x \mp 2)}{p}\right)=\left(\frac{2}{p}\right)\left(\sum_{x=0}^{p-1}\left(\frac{x \mp 2}{p}\right)-\left(\frac{\mp 3}{p}\right)\right)=-\left(\frac{\mp 6}{p}\right) .
\end{aligned}
$$

Thus applying (2.4) and the fact $P_{n}( \pm 1)=( \pm 1)^{n}$ (see [6]) we deduce the result.

Now assume $t \not \equiv \pm 1(\bmod p)$. For $A, B, C \in \mathbb{Z}_{p}$, it is easily seen that (see for example [12, pp. 221-222])

$$
\# E_{p}\left(y^{2}=x^{3}+A x^{2}+B x+C\right)=p+1+\sum_{x=0}^{p-1}\left(\frac{x^{3}+A x^{2}+B x+C}{p}\right) .
$$

Taking $\lambda=(1-t) / 2$ in Lemma 2.6 and applying the above and (2.1) we see that

$$
\begin{aligned}
P_{\frac{p-1}{2}}(t) & =\sum_{k=0}^{\frac{p-1}{2}}\left(\begin{array}{c}
\frac{p-1}{2}+k \\
k
\end{array}\right)\left(\begin{array}{c}
\frac{p-1}{2} \\
k
\end{array}\right)\left(\frac{t-1}{2}\right)^{k} \\
& \equiv(-1)^{\frac{p-1}{2}}\left(p+1-\# E_{p}\left(y^{2}=x(x-1)(x-(1-t) / 2)\right)\right) \\
& =(-1)^{\frac{p+1}{2}} \sum_{x=0}^{p-1}\left(\frac{x(x-1)(x-(1-t) / 2)}{p}\right)(\bmod p) .
\end{aligned}
$$

Since

$$
\begin{aligned}
& \sum_{x=0}^{p-1}\left(\frac{x(x-1)(x-(1-t) / 2)}{p}\right) \\
& =\sum_{x=0}^{p-1}\left(\frac{\frac{x}{2}\left(\frac{x}{2}-1\right)\left(\frac{x}{2}-\frac{1-t}{2}\right)}{p}\right)=\left(\frac{2}{p}\right) \sum_{x=0}^{p-1}\left(\frac{x(x-2)(x+t-1)}{p}\right) \\
& =\left(\frac{2}{p}\right) \sum_{x=0}^{p-1}\left(\frac{x^{3}+(t-3) x^{2}-2(t-1) x}{p}\right) \\
& =\left(\frac{2}{p}\right) \sum_{x=0}^{p-1}\left(\frac{\left(x-\frac{t-3}{3}\right)^{3}+(t-3)\left(x-\frac{t-3}{3}\right)^{2}-2(t-1)\left(x-\frac{t-3}{3}\right)}{p}\right) \\
& =\left(\frac{2}{p}\right) \sum_{x=0}^{p-1}\left(\frac{x^{3}-\frac{t^{2}+3}{3} x+\frac{2 t^{3}-18 t}{27}}{p}\right)=\left(\frac{2}{p}\right) \sum_{x=0}^{p-1}\left(\frac{\left(\frac{x}{3}\right)^{3}-\frac{t^{2}+3}{3} \cdot \frac{x}{3}+\frac{2 t^{3}-18 t}{27}}{p}\right) \\
& =\left(\frac{6}{p}\right) \sum_{x=0}^{p-1}\left(\frac{x^{3}-3\left(t^{2}+3\right) x+2 t\left(t^{2}-9\right)}{p}\right),
\end{aligned}
$$

by the above and (2.4) we obtain the result. The proof is now complete. 
Theorem 2.12. Let $p>3$ be a prime. Then

$$
\begin{aligned}
& P_{\frac{p-1}{2}}(-31) \equiv \sum_{k=0}^{\frac{p-1}{2}}\left(\begin{array}{c}
2 k \\
k
\end{array}\right)^{2} \equiv \sum_{k=0}^{\frac{p-1}{2}} \frac{\left(\begin{array}{c}
2 k \\
k
\end{array}\right)^{2}}{256^{k}} \equiv-\left(\frac{p}{3}\right) \sum_{x=0}^{p-1}\left(\frac{x^{3}-723 x-7378}{p}\right)(\bmod p), \\
& P_{\frac{p-1}{2}}(33) \\
& \equiv \sum_{k=0}^{\frac{p-1}{2}}(-1)^{k}\left(\begin{array}{c}
2 k \\
k
\end{array}\right)^{2} \equiv(-1)^{\frac{p-1}{2}} \sum_{k=0}^{\frac{p-1}{2}} \frac{\left(\begin{array}{c}
2 k \\
k
\end{array}\right)^{2}}{(-256)^{k}} \equiv(-1)^{\frac{p+1}{2}} \sum_{x=0}^{p-1}\left(\frac{x^{3}-91 x+330}{p}\right)(\bmod p), \\
& P_{\frac{p-1}{2}}(-15) \equiv \sum_{k=0}^{\frac{p-1}{2}} \frac{\left(\begin{array}{c}
2 k \\
k
\end{array}\right)^{2}}{2^{k}} \equiv\left(\frac{2}{p}\right) \sum_{k=0}^{\frac{p-1}{2}} \frac{\left(\begin{array}{c}
k \\
k
\end{array}\right)^{2}}{128^{k}} \equiv(-1)^{\frac{p+1}{2}} \sum_{x=0}^{p-1}\left(\frac{x^{3}-19 x-30}{p}\right)(\bmod p), \\
& P_{\frac{p-1}{2}}(9) \equiv \sum_{k=0}^{\frac{p-1}{2}} \frac{\left(\begin{array}{c}
2 k \\
k
\end{array}\right)^{2}}{(-4)^{k}} \equiv(-1)^{\frac{p-1}{2}} \sum_{k=0}^{\frac{p-1}{2}} \frac{\left(\begin{array}{c}
2 k \\
k
\end{array}\right)^{2}}{(-64)^{k}} \equiv(-1)^{\frac{p+1}{2}} \sum_{x=0}^{p-1}\left(\frac{x^{3}-7 x+6}{p}\right)(\bmod p), \\
& P_{\frac{p-1}{2}}(5) \equiv \sum_{k=0}^{\frac{p-1}{2}} \frac{\left(\begin{array}{c}
2 k \\
k
\end{array}\right)^{2}}{(-8)^{k}} \equiv\left(\frac{-2}{p}\right) \sum_{k=0}^{\frac{p-1}{2}} \frac{\left(\begin{array}{c}
2 k \\
k
\end{array}\right)^{2}}{(-32)^{k}} \equiv-\left(\frac{p}{3}\right) \sum_{x=0}^{p-1}\left(\frac{x^{3}-21 x+20}{p}\right)(\bmod p) .
\end{aligned}
$$

Proof. Taking $t=-31$ in Theorem 2.11 and applying Theorem 2.7 we see that

$$
\begin{aligned}
P_{\frac{p-1}{2}}(-31) & \equiv \sum_{k=0}^{\frac{p-1}{2}}\left(\begin{array}{c}
2 k \\
k
\end{array}\right)^{2} \equiv \sum_{k=0}^{\frac{p-1}{2}} \frac{\left(\begin{array}{c}
2 k \\
k
\end{array}\right)^{2}}{256^{k}} \equiv-\left(\frac{-6}{p}\right) \sum_{x=0}^{p-1}\left(\frac{x^{3}-3\left(31^{2}+3\right) x-62\left(31^{2}-9\right)}{p}\right) \\
& =-\left(\frac{-6}{p}\right) \sum_{x=0}^{p-1}\left(\frac{(2 x)^{3}-4 \cdot 723 \cdot 2 x-8 \cdot 7378}{p}\right) \\
& =-\left(\frac{-3}{p}\right) \sum_{x=0}^{p-1}\left(\frac{x^{3}-723 x-7378}{p}\right)(\bmod p) .
\end{aligned}
$$

Taking $t=33$ in Theorem 2.11 and applying Theorem 2.7 we see that

$$
\begin{aligned}
& P_{\frac{p-1}{2}}(33) \\
& \equiv \sum_{k=0}^{\frac{p-1}{2}}(-1)^{k}\left(\begin{array}{c}
2 k \\
k
\end{array}\right)^{2} \equiv\left(\frac{-16}{p}\right) \sum_{k=0}^{\frac{p-1}{2}} \frac{\left(\begin{array}{c}
2 k \\
k
\end{array}\right)^{2}}{(-256)^{k}} \equiv-\left(\frac{-6}{p}\right) \sum_{x=0}^{p-1}\left(\frac{x^{3}-3276 x+71280}{p}\right) \\
& =-\left(\frac{-6}{p}\right) \sum_{x=0}^{p-1}\left(\frac{(6 x)^{3}-36 \cdot 91 \cdot 6 x+216 \cdot 330}{p}\right) \\
& =-\left(\frac{-1}{p}\right) \sum_{x=0}^{p-1}\left(\frac{x^{3}-91 x+330}{p}\right)(\bmod p) .
\end{aligned}
$$

The remaining congruences can be proved similarly.

For $a, b, n \in \mathbb{N}$, if $n=a x^{2}+b y^{2}$ for some $x, y \in \mathbb{Z}$, we say that $n=a x^{2}+b y^{2}$. In 2003, Rodriguez-Villegas [1] posed many conjectures on supercongruences. In particular, he conjectured that for any prime $p>3$,

$$
\sum_{k=0}^{p-1} \frac{(4 k) !}{256^{k} k !^{4}} \equiv \begin{cases}4 x^{2}-2 p\left(\bmod p^{2}\right) & \text { if } p \equiv 1,3(\bmod 8), \text { and so } p=x^{2}+2 y^{2}, \\ 0\left(\bmod p^{2}\right) & \text { if } p \equiv 5,7(\bmod 8)\end{cases}
$$


and

$$
\sum_{k=0}^{p-1} \frac{\left(\begin{array}{c}
2 k \\
k
\end{array}\right)^{2}\left(\begin{array}{c}
3 k \\
k
\end{array}\right)}{108^{k}} \equiv \begin{cases}4 x^{2}-2 p\left(\bmod p^{2}\right) & \text { if } 3 \mid p-1, \text { and so } p=x^{2}+3 y^{2} \\
0\left(\bmod p^{2}\right) & \text { if } 3 \mid p-2 .\end{cases}
$$

Recently Zhi-Wei Sun ([13, 14]) made a lot of conjectures on supercongruences. In particular, he conjectured that for a prime $p \neq 2,7$,

$$
\sum_{k=0}^{p-1}\left(\begin{array}{c}
2 k \\
k
\end{array}\right)^{3} \equiv \sum_{k=0}^{p-1} \frac{(4 k) !}{81^{k} k !^{4}} \equiv \begin{cases}4 x^{2}-2 p\left(\bmod p^{2}\right) & \text { if }\left(\frac{p}{7}\right)=1 \text { and so } p=x^{2}+7 y^{2} \\
0\left(\bmod p^{2}\right) & \text { if }\left(\frac{p}{7}\right)=-1\end{cases}
$$

Inspired by his work, we pose the following conjectures.

Conjecture 2.1. Let $p>3$ be a prime. Then

$\sum_{k=0}^{p-1} \frac{(4 k) !}{648^{k} k !^{4}} \equiv \begin{cases}4 x^{2}-2 p\left(\bmod p^{2}\right) & \text { if } p \equiv 1(\bmod 4) \text { and } p=x^{2}+y^{2} \text { with } 2 \nmid x, \\ 0\left(\bmod p^{2}\right) & \text { if } p \equiv 3(\bmod 4) .\end{cases}$

Conjecture 2.2. Let $p>3$ be a prime. Then

$$
\sum_{k=0}^{p-1} \frac{(4 k) !}{(-144)^{k} k !^{4}} \equiv \begin{cases}4 x^{2}-2 p\left(\bmod p^{2}\right) & \text { if } p \equiv 1(\bmod 3), \text { and so } p=x^{2}+3 y^{2}, \\ 0\left(\bmod p^{2}\right) & \text { if } p \equiv 2(\bmod 3) .\end{cases}
$$

Conjecture 2.3. Let $p \neq 2,3,7$ be a prime. Then

$$
\sum_{k=0}^{p-1} \frac{(4 k) !}{(-3969)^{k} k !^{4}} \equiv \begin{cases}4 x^{2}-2 p\left(\bmod p^{2}\right) & \text { if } p \equiv 1,2,4(\bmod 7), \text { and so } p=x^{2}+7 y^{2} \\ 0\left(\bmod p^{2}\right) & \text { if } p \equiv 3,5,6(\bmod 7) .\end{cases}
$$

Conjecture 2.4. Let $p \neq 2,3,11$ be a prime. Then

$$
\sum_{k=0}^{p-1} \frac{(6 k) !}{66^{3 k}(3 k) ! k !^{3}} \equiv \begin{cases}\left(\frac{p}{33}\right)\left(4 x^{2}-2 p\right)\left(\bmod p^{2}\right) & \text { if } 4 \mid p-1 \text { and } p=x^{2}+y^{2} \text { with } 2 \nmid x, \\ 0\left(\bmod p^{2}\right) & \text { if } 4 \mid p-3 .\end{cases}
$$

Conjecture 2.5. Let $p>5$ be a prime. Then

$$
\sum_{k=0}^{p-1} \frac{(6 k) !}{20^{3 k}(3 k) ! k !^{3}} \equiv \begin{cases}\left(\frac{-5}{p}\right)\left(4 x^{2}-2 p\right)\left(\bmod p^{2}\right) & \text { if } p \equiv 1,3(\bmod 8) \text { and } p=x^{2}+2 y^{2} \\ 0\left(\bmod p^{2}\right) & \text { if } p \equiv 5,7(\bmod 8) .\end{cases}
$$

Conjecture 2.6. Let $p>5$ be a prime. Then

$$
\sum_{k=0}^{p-1} \frac{(6 k) !}{54000^{k}(3 k) ! k !^{3}} \equiv \begin{cases}\left(\frac{p}{5}\right)\left(4 x^{2}-2 p\right)\left(\bmod p^{2}\right) & \text { if } 3 \mid p-1, \text { and so } p=x^{2}+3 y^{2} \\ 0\left(\bmod p^{2}\right) & \text { if } 3 \mid p-2 .\end{cases}
$$

Conjecture 2.7. Let $p>5$ be a prime. Then

$$
\begin{aligned}
& \sum_{k=0}^{p-1} \frac{(6 k) !}{(-12288000)^{k}(3 k) ! k !^{3}} \\
& \equiv \begin{cases}\left(\frac{10}{p}\right)\left(L^{2}-2 p\right)\left(\bmod p^{2}\right) & \text { if } p \equiv 1(\bmod 3), \text { and } s o 4 p=L^{2}+27 M^{2}, \\
0\left(\bmod p^{2}\right) & \text { if } p \equiv 2(\bmod 3) .\end{cases}
\end{aligned}
$$

Conjecture 2.8. Let $p>7$ be a prime. Then

$$
\sum_{k=0}^{p-1} \frac{(6 k) !}{(-15)^{3 k}(3 k) ! k !^{3}} \equiv \begin{cases}\left(\frac{p}{15}\right)\left(4 x^{2}-2 p\right)\left(\bmod p^{2}\right) & \text { if }\left(\frac{p}{7}\right)=1 \text {, and so } p=x^{2}+7 y^{2}, \\ 0\left(\bmod p^{2}\right) & \text { if }\left(\frac{p}{7}\right)=-1 .\end{cases}
$$


Conjecture 2.9. Let $p \neq 2,3,5,7,17$ be a prime. Then

$\sum_{k=0}^{p-1} \frac{(6 k) !}{255^{3 k}(3 k) ! k !^{3}} \equiv \begin{cases}\left(\frac{p}{255}\right)\left(4 x^{2}-2 p\right)\left(\bmod p^{2}\right) & \text { if }\left(\frac{p}{7}\right)=1 \text {, and so } p=x^{2}+7 y^{2}, \\ 0\left(\bmod p^{2}\right) & \text { if }\left(\frac{p}{7}\right)=-1 .\end{cases}$

Conjecture 2.10. Let $p>3$ be a prime. Then

$$
\sum_{k=0}^{p-1} \frac{\left(\begin{array}{c}
2 k \\
k
\end{array}\right)^{2}\left(\begin{array}{c}
3 k \\
k
\end{array}\right)}{1458^{k}} \equiv \begin{cases}4 x^{2}-2 p\left(\bmod p^{2}\right) & \text { if } p \equiv 1(\bmod 3), \text { and so } p=x^{2}+3 y^{2}, \\
0\left(\bmod p^{2}\right) & \text { if } p \equiv 2(\bmod 3) .\end{cases}
$$

Conjecture 2.11. Let $p>5$ be a prime. Then

$$
\sum_{k=0}^{p-1} \frac{\left(\begin{array}{c}
2 k \\
k
\end{array}\right)^{2}\left(\begin{array}{c}
3 k \\
k
\end{array}\right)}{15^{3 k}} \equiv \begin{cases}4 x^{2}-2 p\left(\bmod p^{2}\right) & \text { if } p \equiv 1,4(\bmod 15), \text { and } \text { so } p=x^{2}+15 y^{2}, \\
2 p-12 x^{2}\left(\bmod p^{2}\right) & \text { if } p \equiv 2,8(\bmod 15), \text { and } s o p=3 x^{2}+5 y^{2}, \\
0\left(\bmod p^{2}\right) & \text { if } p \equiv 7,11,13,14(\bmod 15) .\end{cases}
$$

Conjecture 2.12. Let $p>5$ be a prime. Then

$$
\sum_{k=0}^{p-1} \frac{\left(\begin{array}{c}
2 k \\
k
\end{array}\right)^{2}\left(\begin{array}{c}
3 k \\
k
\end{array}\right)}{(-8640)^{k}}=\left\{\begin{array}{lc}
4 x^{2}-2 p\left(\bmod p^{2}\right) & \text { if } 3 \mid p-1, p=x^{2}+3 y^{2} \text { and } 5 \mid x y, \\
p-2 x^{2} \pm 6 x y\left(\bmod p^{2}\right) & \text { if } 3 \mid p-1, p=x^{2}+3 y^{2}, 5 \nmid x y \\
& \text { and } x \equiv \pm y, \pm 2 y(\bmod 5), \\
0\left(\bmod p^{2}\right) & \text { if } 3 \mid p-2 .
\end{array}\right.
$$

Conjecture 2.13. Let $p>3$ be a prime. Then

$$
\sum_{k=0}^{p-1} \frac{(3 k) !}{54^{k} \cdot k !^{3}} \equiv \begin{cases}\left(\frac{x}{3}\right)\left(2 x-\frac{p}{2 x}\right)\left(\bmod p^{2}\right) & \text { if } 3 \mid p-1, \text { and so } p=x^{2}+3 y^{2}, \\ 0\left(\bmod p^{2}\right) & \text { if } 3 \mid p-2 .\end{cases}
$$

\section{REFERENCES}

1. S. Ahlgren and K. Ono, A Gaussian hypergeometric series evaluation and Apéry number congruences, J. Reine Angew. Math. 518 (2000), 187-212. MR1739404 (2001c:11057)

2. B.C. Berndt, R.J. Evans and K.S. Williams, Gauss and Jacobi Sums, John Wiley \& Sons, New York, 1998. MR1625181 (99d:11092)

3. S. Chowla, B. Dwork and R. J. Evans, On the $\bmod p^{2}$ determination of $\left(\begin{array}{c}(p-1) / 2 \\ (p-1) / 4\end{array}\right)$, J. Number Theory 24 (1986), 188-196. MR863654 (88a:11130)

4. H.W. Gould, Combinatorial Identities, A Standardized Set of Tables Listing 500 Binomial Coefficient Summations, Morgantown, W. Va., 1972. MR0354401(50:6879)

5. P.-R. Loh and R.C. Rhoades, p-adic and combinatorial properties of modular form coefficients, Int. J. Number Theory 2 (2006), 305-328. MR2240232 (2007f:11047)

6. W. Magnus, F. Oberhettinger and R.P. Soni, Formulas and Theorems for the Special Functions of Mathematical Physics, 3rd. ed., D. Grund. Math. Wissen. Springer, New York, 1966, pp. 228-232. MR0232968 (38:1291)

7. E. Mortenson, A supercongruence conjecture of Rodriguez-Villegas for a certain truncated hypergeometric function, J. Number Theory 99 (2003), 139-147. MR1957248(2004e:11089)

8. E. Mortenson, Supercongruences between truncated ${ }_{2} F_{1}$ by hypergeometric functions and their Gaussian analogs, Trans. Amer. Math. Soc. 355 (2003), 987-1007. MR.1938742(2003i:11119)

9. E. Mortenson, Supercongruences for truncated ${ }_{n+1} F_{n}$ hypergeometric series with applications to certain weight three newforms, Proc. Amer. Math. Soc. 133 (2005), 321-330. MR2093051 (2005f:11080)

10. K. Ono, The Web of Modularity: Arithmetic of the Coefficients of Modular Forms and q-series, CBMS Monograph 102, Amer. Math. Soc., Providence, RI, 2004. MR2020489 (2005c:11053)

11. F. Rodriguez-Villegas, Hypergeometric families of Calabi-Yau manifolds. Calabi-Yau Varieties and Mirror Symmetry (Yui, Noriko (ed.) et al., Toronto, ON, 2001), 223-231, Fields Inst. Commun., 38, Amer. Math. Soc., Providence, RI, 2003. MR2019156 (2005b:11086) 
12. Z.H. Sun, On the number of incongruent residues of $x^{4}+a x^{2}+b x$ modulo $p$, J. Number Theory 119 (2006), 210-241. MR.2250045 (2007f:11003)

13. Z.W. Sun, On congruences related to central binomial coefficients, preprint, arXiv:0911.2415, http://arxiv.org/abs/0911.2415.

14. Z.W. Sun, Open conjectures on congruences, arXiv:0911.5665. http://arxiv.org/abs/ 0911.5665 .

15. L. Van Hamme, Proof of a conjecture of Beukers on Apéry numbers, Proceedings of the Conference on p-adic Analysis (N. De Grande-De Kimpe and L. Van Hamme, ed., Houthalen, 1987), pp. 189-195, Vrije Univ. Brussel, Brussels, 1986. MR921871 (89b:11007)

School of the Mathematical Sciences, Huaiyin Normal University, Huaian, Jiangsu 223001, People's Republic of China

E-mail address: szh6174@yahoo.com 